\title{
Discontinuous Galerkin finite element scheme for a conserved higher-order traffic flow model by exploring Riemann solvers
}

\author{
Dian-liang Qiao ${ }^{\mathrm{a}, \mathrm{b}}$, Peng Zhang ${ }^{\mathrm{a}, \mathrm{e}, *}$, S.C. Wong ${ }^{\mathrm{c}}, \mathrm{Keechoo} \mathrm{Choi}^{\mathrm{d}}$ \\ ${ }^{a}$ Shanghai Institute of Applied Mathematics and Mechanics, Shanghai University, \\ Shanghai, P.R. China \\ ${ }^{b}$ Shanghai Xinqiao Polytechnic college, Shanghai P.R. China \\ ${ }^{c}$ Department of Civil Engineering, The University of Hong Kong, Pokfulam Road, Hong \\ Kong SAR, P.R. China \\ ${ }^{d}$ Department of Transportation Engineering, TOD-based Sustainable Urban \\ Transportation Center, Ajou University, Korea \\ ${ }^{e}$ Shanghai Key Laboratory of Mechanics in Energy Engineering
}

\begin{abstract}
The discontinuous Galerkin (DG) scheme is used to solve a conserved higherorder $(\mathrm{CHO})$ traffic flow model by exploring several Riemann solvers. The second-order accurate DG scheme is found to be adequate in that the accuracy is comparable to the weighted essentially non-oscillatory (WENO) scheme with fifth-order accuracy and much better than the scheme with first-order accuracy in resolving a wide moving jam with a shock profile. Moreover, it considerably reduces the differences between the proposed solvers in generating numerical viscosities or errors. Thus, this scheme can maintain high efficiency when a simple solver is adopted. The scheme could be extended to solve more complex problems, such as those related to traffic flow in a network.
\end{abstract}

Keywords: Monotone numerical fluxes; DG scheme; WENO scheme; Shock; Wide moving jam

\footnotetext{
*Corresponding author.

Email address: pzhang@mail.shu.edu.cn (Peng Zhang)
} 


\section{Introduction}

A Riemann solver, or numerical flux, is sufficient for designing a first-order accurate scheme for hyperbolic conservation laws. The Godunov, EngquistOsher (EO), and Lax-Friedrichs (LF) are well-known numerical fluxes for solving scalar equations, such as the Lighthill-Whitham-Richards (LWR) model $[16,21]$ and the Burgers equation, which give rise, respectively, to the Godunov, EO, and LF schemes [24, 4, 23, 6] (see also [8, 9, 29, 5, 3, $2,15,11,26,35]$ for studies of the LWR or Multi-class LWR models). To solve the conserved higher-order ( $\mathrm{CHO}$ ) model [33], which is an extension of the LWR model, the aforementioned fluxes were extended with a so-called traffic flow (TF) flux to design a number of first-order accurate schemes [30]. Although these extended fluxes were able to reproduce stable and convergent numerical solutions, considerable numerical viscosities or errors were observed.

This sequence simplifies the expression of these fluxes and the computation becomes more efficient; however, the shock profile of the reproduced wide moving jam in the $\mathrm{CHO}$ model becomes smoother and the backward moving wave becomes faster, which suggests an increase in numerical viscosity. It seems that the improvement in efficiency from computing a simpler numerical flux is counteracted by an increase in numerical viscosity. If a firstorder accurate scheme is adopted, the grid needs to be refined to improve the solution. Generally, improving the scheme's accuracy should suppress the redundant numerical viscosity in a simpler flux. More precisely, numerical fluxes (either simple or complicated) are expected to produce similarly accurate solutions in a higher-order scheme. In this case, using a simpler flux will improve the scheme's efficiency.

Following this insight, this paper designs a discontinuous Galerkin (DG) scheme for solving the $\mathrm{CHO}$ model by exploiting the numerical fluxes extended from the Godunov, EO, LF and TF fluxes [30, 19]. The DG scheme is found to be more accurate than the first-order scheme in that it is able to suppress differences between the explored solvers when generating numerical viscosities. Moreover, we find that the performance of the second-order DG scheme is similar to that of the fifth-order WENO schemes, a similarity that is due to the existence of a shock profile in the solution, although the latter scheme is expected to be more accurate in generating smooth solutions. These findings indicate that the second-order DG scheme is adequate for the model, especially when a simpler flux with a lower computational cost is 
adopted. To enhance the argument, an analytical solution for a wide moving jam is constructed for comparison with the numerical solutions.

The remainder of this paper is organized as follows. Section 2 discusses the $\mathrm{CHO}$ model, together with its solution for a wide moving jam. In Section 3, the extended Godunov, EO, LF and TF numerical fluxes are derived by applying the Riemann solution to the model's homogeneous system. In Section 4, the DG schemes are constructed using these fluxes. In Section 5, numerical solutions to the wide moving jam are derived through simulation, and the solutions produced by the first-order accurate scheme, the fifth-order accurate WENO scheme and the second-order accurate DG scheme are compared with the analytical solution. Section 6 concludes the paper.

\section{Model equations}

Taken as a continuum, the mass conservation of traffic flow is described through the following partial differential equation:

$$
\partial_{t} \rho+\partial_{x}(\rho v)=0
$$

where $\rho(x, t)$ and $v(x, t)$ are the density and velocity in location $x$ at time $t$, respectively, and $q(x, t)=\rho(x, t) v(x, t)$ denotes the flow at $(x, t)$. Assume that there is a determined equilibrium velocity-density relationship $v=v_{e}(\rho)$ $\left(v_{e}^{\prime}(\rho)<0\right)$. Then, the substitution of $v$ in Eq. (1) by this equation gives rise to the following well-known LWR model [16, 21]:

$$
\partial_{t} \rho+\partial_{x}\left(\rho v_{e}(\rho)\right)=0
$$

The traffic flow model has been improved since the LWR model was first proposed. Among the improved models, the higher-order model [20, 10, $18,25]$ is typically used to describe stop-and-go waves under a metastable mechanism.

\subsection{Conserved higher-order model}

Zhang et al. [33] proposed a conserved higher-order (CHO) model with the novel introduction of pseudo-density $w(x, t)$, which is transformed from the velocity through the function $v=V(w)$, where $V(\cdot)$ is also taken as a velocity-density relationship with $V^{\prime}(w)<0$, and $(w V(w))^{\prime \prime}<0$. Substituting the velocity $v$ with $V(w)$ in Eq. (1) and an assumed acceleration 
equation, the $\mathrm{CHO}$ model can be written as the following conserved system:

$$
\begin{aligned}
& \partial_{t} \rho+\partial_{x}(\rho V(w))=0, \\
& \partial_{t} w+\partial_{x}(w V(w))=\beta^{-1}\left(V(w)-v_{e}(\rho)\right),
\end{aligned}
$$

where Eq. (4) can be viewed as a pseudo-mass conservation with relaxation, and $\beta$ is associated with the relaxation time $\tau$ through $\beta=-\tau V^{\prime}(w)>0$. Eq. (4) can also be rewritten in the following transport form [33, 30]:

$$
\partial_{t}(\rho z)+\partial_{x}(\rho z v)=\beta^{-1}\left(V(\rho z)-v_{e}(\rho)\right),
$$

where $\rho z$ looks like a "momentum" that should be conserved, and the ratio $z=w / \rho$ reflects the deviation of some ideally allowed density $w$ (or headway $1 / w$ ) from the actual vehicular density $\rho$ (or headway $1 / \rho$ ). See also $[13,14]$ for a similar formulation and interpretation.

Remarkably, the system takes an elegant equation form, which is consistent with the LWR model, such that Eqs (2)-(4) become equivalent in the sense that they produce a weak solution when setting $w \equiv \rho$ and $V(w) \equiv$ $v_{e}(\rho)$. This suggests that in general, studying the analytical properties of the system should be much easer than it is for other higher-order models, yet the system is able to reproduce similar phenomena, such as stop-and-go waves, under the same metastable mechanism. See [33] for detailed discussions of the hyperbolicity and the physically bounded solution of system (3)-(4).

\subsection{Solution for a wide moving jam}

Zhang and his collaborators proposed an analytical technique to determine the characteristic parameters of a wide moving jam solution in higherorder models $[31,32,34,28]$. The same technique is adopted to deal with a similar solution in system (3)-(4) (see also [7, 22] for similar techniques). Fig. 1(a) shows the profile of the wide moving jam, which is composed of a shock (the upstream front) and a smooth transition layer (the downstream front). We assume that both fronts are connected approximately by two equilibrium states, $\left(\rho_{A}, q_{e}\left(\rho_{A}\right)\right)$ and $\left(\rho_{B}, q_{e}\left(\rho_{B}\right)\right)$, in the flow-density phase plane (Fig. 1(b)). Here, the fundamental diagram $q_{e}(\rho) \equiv \rho v_{e}(\rho)$ is shown as a non-convex function, and an equilibrium state is defined as a constant solution by which the relaxation term in Eq. (4) vanishes. This suggests that the aforementioned two equilibrium states correspond to $w_{A}$ and $w_{B}$, which are determined by $V\left(w_{A}\right)=v_{e}\left(\rho_{A}\right)$ and $V\left(w_{B}\right)=v_{e}\left(\rho_{B}\right)$. 

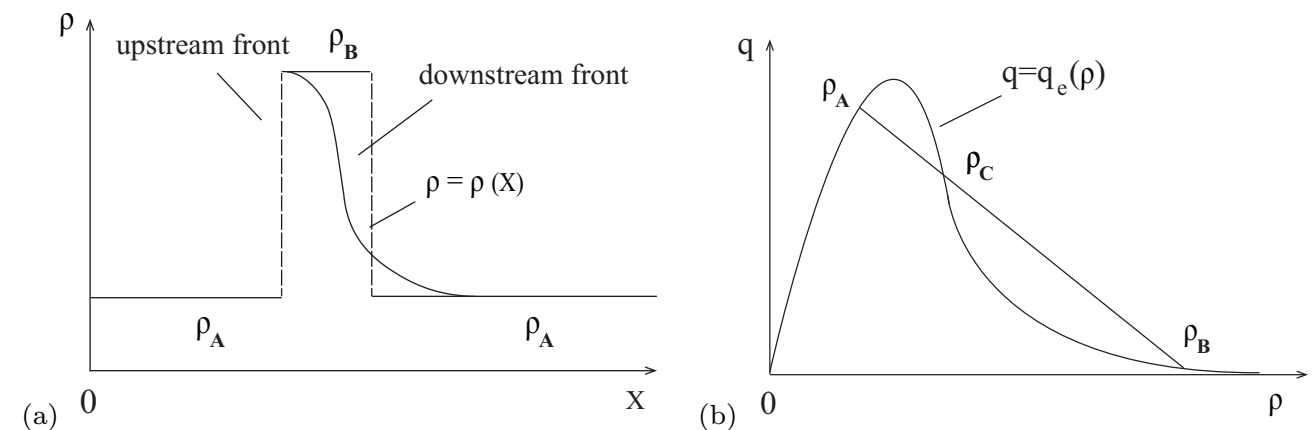

Fig. 1-(a) A wide moving jam composed of a shock wave (the upstream front) and a smooth transition layer (the downstream front); (b) the corresponding phase-plot in the density-flow plane.

To describe the upstream front or shock that is discontinuous with a jump from $\rho_{A}$ to $\rho_{B}$, the Rankine-Hugnoniot (R-H) conditions are applied to the conservative system (3)-(4). Thus, we have

$$
a=\frac{\rho_{A} v_{e}\left(\rho_{A}\right)-\rho_{B} v_{e}\left(\rho_{B}\right)}{\rho_{A}-\rho_{B}}=\frac{w_{A} v_{e}\left(\rho_{A}\right)-w_{B} v_{e}\left(\rho_{B}\right)}{w_{A}-w_{B}},
$$

where $a<0$ is the shock speed. In Fig. 1(b), the shock is represented by the jump from $\left(\rho_{A}, q_{e}\left(\rho_{A}\right)\right)$ to $\left(\rho_{B}, q_{e}\left(\rho_{B}\right)\right)$.

To describe the downstream front or transition layer that smoothly links $\rho_{A}$ to $\rho_{B}$, we assume a smooth traveling wave solution $(\rho, w)=(\rho(x-$ at), $w(x-a t))$ with the same wave speed $a<0$. Substituting this into system (3)-(4), we have

$$
\frac{d \rho}{d X}=\frac{\rho V^{\prime}(w)}{\beta q_{0}} \frac{a \rho+q_{0}-q_{e}(\rho)}{a-V(w)-w V^{\prime}(w)},
$$

where $X=x-a t, q_{0}$ is the integral constant and $w$ is determined as an implicit function of $\rho$ through

$$
V(w)=a+q_{0} / \rho \geq 0 .
$$

Equation (8) can be rewritten as $q=a \rho+q_{0}$, which suggests that the transition layer is represented by the segment between $\left(\rho_{A}, q_{e}\left(\rho_{A}\right)\right)$ and $\left(\rho_{B}, q_{e}\left(\rho_{B}\right)\right)$ in Fig. 1(b). Fig. 1(a) shows that $\rho(X)$ is strictly decreasing with $X$, thus the term on the right side of Eq. (7) is required to be negative. As Fig. 1(b) 
shows, $a \rho+q_{0}-q_{e}(\rho) \lessgtr 0$, for $\rho \lessgtr \rho_{C}$, where $\left(\rho_{C}, q_{e}\left(\rho_{C}\right)\right)$ is intersected by the segment $q=a \rho+q_{0}$ and the fundamental diagram $q=q_{e}(\rho)$, with

$$
a \rho_{C}+q_{0}=q_{e}\left(\rho_{C}\right) .
$$

Recall that $\beta>0$ and $V^{\prime}(w)<0$, and Eq. (8) implies that $q_{0}>0$ because $a<0$. Then, we should have $a-V(w)-w V^{\prime}(w) \lessgtr 0$, for $\rho \lessgtr \rho_{C}$. This requirement can be satisfied because Eq. (8) implies that $w$ is strictly increasing with $\rho$ and $a-V(w)-w V^{\prime}(w)$ is strictly increasing with $w$, thus $\left(a-V(w)-w V^{\prime}(w)\right)^{\prime}=-(w V(w))^{\prime \prime}>0$. This discussion also implies that

$$
a=V\left(w_{C}\right)+w_{C} V^{\prime}\left(w_{C}\right),
$$

where $w_{C}$ is associated with $\rho_{C}$ through $V\left(w_{C}\right)=v_{e}\left(\rho_{C}\right)$.

Summarizing the discussion of Eqs. (6) to (10), we have the following three algebraic equations.

$-w_{C} V^{\prime}\left(w_{C}\right)=\frac{\rho_{A} \rho_{B}\left(v_{e}\left(\rho_{A}\right)-v_{e}\left(\rho_{B}\right)\right.}{\rho_{C}\left(\rho_{B}-\rho_{A}\right)}=v_{e}\left(\rho_{C}\right)-\frac{q_{e}\left(\rho_{A}\right)-q_{e}\left(\rho_{B}\right)}{\rho_{A}-\rho_{B}}, \frac{w_{A}}{\rho_{A}}=\frac{w_{B}}{\rho_{B}}$,

for solving $\rho_{A}, \rho_{B}$, and $\rho_{C}$, where $w_{\chi}$ is associated with $\rho_{\chi}$ through the function $w=V^{-1}\left[v_{e}(\rho)\right]$. The wave speed is determined by Eq. (10).

\section{Riemann solvers for the homogeneous system}

System (3)-(4) is rewritten in the following vector form:

$$
\mathbf{u}_{t}+\mathbf{f}(\mathbf{u})_{x}=\mathbf{s}(\mathbf{u})
$$

with $\mathbf{u}=(\rho, w)^{T}, \mathbf{f}(\mathbf{u})=\left(f_{1}(\mathbf{u}), f_{2}(\mathbf{u})\right)^{T}, f_{1}(\mathbf{u})=\rho V(w), f_{2}(\mathbf{u})=w V(w)$ and $\mathbf{s}(\mathbf{u})=\left(0, \beta^{-1}\left(V(w)-v_{e}(\rho)\right)\right)^{T}$. To derive a numerical flux, the Riemann problem in the homogeneous system

$$
\mathbf{u}_{t}+\mathbf{f}(\mathbf{u})_{x}=0
$$

is of interest. The problem is actually set with the initial values

$$
\mathbf{u}(x, 0)= \begin{cases}\mathbf{u}_{1}, & x<0 \\ \mathbf{u}_{2}, & x>0\end{cases}
$$

where $\mathbf{u}(x, 0)=(\rho(x, 0), w(x, 0))^{T}, \mathbf{u}_{1}=\left(\rho_{1}, w_{1}\right)^{T}$ and $\mathbf{u}_{2}=\left(\rho_{2}, w_{2}\right)^{T}$. 


\subsection{Exact Riemann solver or the Godunov flux}

The initial values of $(\rho, w)$ are shown in the $x$ - $t$ upper plane (Fig. 2). In the solution, the flow values $\left(f_{1}(\mathbf{u}(0, t)), f_{2}(\mathbf{u}(0, t))\right)$ in the interface $x=0$ are denoted by $\left(\hat{f}_{1}^{G}, \hat{f}_{2}^{G}\right)$, which is the exact Riemann solver that we also call the Godunov flux. This solver is easily derived through the following discussion.

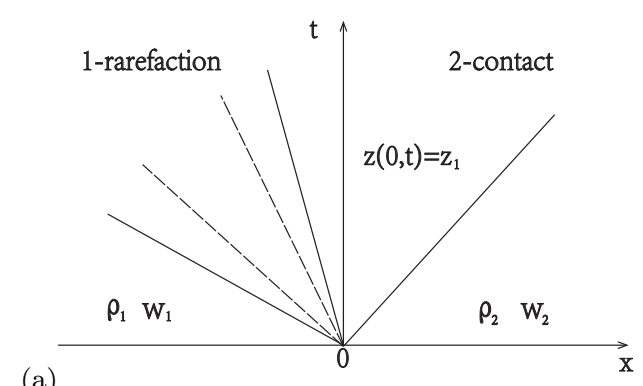

(a)

Fig. 2 Two wave breaking patterns associated with the first characteristic field: (a) the rarefaction wave for $w_{1} \geq w_{2}$; (b) the shock wave for $w_{1}<w_{2}$.

First, the second equation in (13) is independent of $\rho$, which takes the same form as the LWR model and can be rewritten as the characteristic equation

$$
\partial_{t} w+\lambda_{1} \partial w=0
$$

with the eigenvalue $\lambda_{1}=V(w)+w V^{\prime}(w)$. It is obvious that the Riemann problem for the second equation in (13) can be solved independently with

$$
\hat{f}_{2}^{G}=\hat{f}_{2}^{G}\left(w_{1}, w_{2}\right)
$$

where $\hat{f}_{2}^{G}\left(w_{1}, w_{2}\right)$ is the well-known Godunov flux for a scalar conservation law (its expression can be found in Section 3.2). For a $w V(w)$ that is strictly concave with $(w V(w))^{\prime \prime}<0$, the first characteristic field associated with $\lambda_{1}$ or Eq. (14) suggests either a rarefaction or a shock wave, as is shown in Figs $2(\mathrm{a})$ and (b), respectively.

Second, we take the second equation in (13) as a transport form that corresponds to Eq. (5), and multiply this equation by $1 / \rho$ and the first equation in (13) by $z / \rho$. Reducing one of the resultant equations by the other gives another characteristic equation,

$$
\partial_{t} z+\lambda_{2} \partial_{x} z=0
$$


with the eigenvalue $\lambda_{2}=V(w) \geq \lambda_{1}$. Because the characteristic variable $z$ in (16) is a Riemann invariant with respect to the first characteristic field, the value of $z=w / \rho$ does not change through the propagation of the first characteristics in the region $x / t \leq \lambda_{2}\left(w_{2}\right)$, which implies that $z(0, t)=z(x, 0)=z_{1}$. We note that the conclusion is also true for cases involving a shock wave (Fig. $2(\mathrm{~b})$ ), because the second equation in (13) can be written in a transport form corresponding to Eq. (5).

Finally, the two flows at the interface are related by the Riemann invariant $w$ through the relation $f_{1}(\mathbf{u}(0, t))=(z(0, t))^{-1} f_{2}(\mathbf{u}(0, t))=z_{1}^{-1} \hat{f}_{2}^{G}$, so that we have

$$
\hat{f}_{1}^{G}=\frac{\rho_{1}}{w_{1}} \hat{f}_{2}^{G}
$$

The characteristic variable $w$ in Eq. (14) is a Riemann invariant with respect to the second characteristic field, by which we always have a contact discontinuity with the wave speed $\lambda_{2}=V(w)$, and $w$ is continuous across this wave (Figs. 2(a) and (b)). This, together with the aforementioned information, helps to form a complete solution structure for the Riemann problem.

\subsection{Extended Riemann solvers}

The insight for this extension is developed from the relation $f_{1}=z^{-1} f_{2}$, with $z$ being a Riemann invariant. For a numerical flux $\hat{f}_{2}^{\chi}$ that approximates $f_{2}$, the numerical flux that approximates $f_{1}$ is taken as

$$
\hat{f}_{1}^{\chi}=z_{1}^{-1} \hat{f}_{2}^{\chi}, \quad z_{1}=\frac{w_{1}}{\rho_{1}}
$$

Here, $\hat{f}_{2}^{\chi}$ can be taken not only as the Godunov flux $\hat{f}_{2}^{G}$ with $\chi=G$, as in Eq. (17), but also as many other monotone fluxes for the scalar conservation law (see $[24,4,23]$ for the discussion and application of these fluxes). These fluxes, together with the traffic flow (TF) flux for the LWR model (Eq. (2) or the second equation in (13)), are given as follows.

\section{- The Godunov flux:}

$$
\hat{f}_{2}^{G}\left(w_{1}, w_{2}\right)=\left\{\begin{array}{l}
\min _{w_{1} \leq w \leq w_{2}} f_{2}(w), w_{1} \leq w_{2} \\
\max _{w_{1} \geq w \geq w_{2}} f_{2}(w), w_{1}>w_{2}
\end{array}\right.
$$


- The Engquist-Osher flux:

$$
\hat{f}_{2}^{E O}\left(w_{1}, w_{2}\right)=\int_{0}^{w_{1}} \max \left(f_{2}^{\prime}(w), 0\right) d w+\int_{0}^{w_{2}} \min \left(f_{2}^{\prime}(w), 0\right) d w+f_{2}(0) .
$$

- The Lax-Friedrichs flux:

$$
\hat{f}_{2}^{L F}\left(w_{1}, w_{2}\right)=\frac{1}{2}\left[f_{2}\left(w_{1}\right)+f_{2}\left(w_{2}\right)-\alpha\left(w_{2}-w_{1}\right)\right], \alpha=\max _{w}\left|f_{2}^{\prime}(w)\right| .
$$

- The Traffic Flow flux:

$$
\hat{f}_{2}^{T F}\left(w_{1}, w_{2}\right)=w_{1} V\left(w_{2}\right) .
$$

\section{The discontinuous Galerkin finite element scheme}

By dividing the computational interval $(0, L)$ into cells, $I_{i}=\left(x_{i-1 / 2}, x_{i+1 / 2}\right)$, with $\Delta_{i}=x_{i+1 / 2}-x_{i-1 / 2}, i=1, \ldots, N$, we multiply system (12) by a test function $w(x)$ and integrate the resultant equations over $I_{i}$. By applying the integration of the parts to the second term, we have

$$
\begin{gathered}
\int_{I_{i}} \mathbf{u}(x, t)_{t} w(x) d x-\int_{I_{i}} \mathbf{f}(\mathbf{u}) w^{\prime}(x) d x+\mathbf{f}\left(\mathbf{u}\left(x_{i+\frac{1}{2}}, t\right)\right) w\left(x_{i+\frac{1}{2}}\right) \\
-\mathbf{f}\left(\mathbf{u}\left(x_{i-\frac{1}{2}}, t\right)\right) w\left(x_{i-\frac{1}{2}}\right)=\int_{I_{i}} \mathbf{s}(\mathbf{u}) w(x) d x .
\end{gathered}
$$

\subsection{Semi-discrete scheme}

The spatial discretization of the DG finite element method is based on the weak formulation (23) of system (12). Assume that $\mathbf{u}_{h}(x, t)$ is an approximation to $\mathbf{u}(x, t)$, which belongs to the polynomial space $P^{k}\left(I_{i}\right)$ in each cell. Then, $\mathbf{u}_{h}(x, t)$ can be expressed as a linear combination,

$$
\left.\mathbf{u}_{h}(x, t)\right|_{I_{i}}=\sum_{l=0}^{k} \mathbf{u}_{l}^{i}(t) \varphi_{l}^{i}(x),
$$

where $\left\{\varphi_{l}^{i}(x)\right\}_{l=0}^{k}$ is a set of bases of $P^{k}\left(I_{i}\right)$. Take $\left\{\varphi_{l}^{i}(x)\right\}_{l=0}^{k}$ as being orthogonal to each other under the $L^{2}$-norm, that is,

$$
\varphi_{l}^{i}(x)=L_{l}\left(\frac{2\left(x-x_{i}\right)}{\Delta_{i}}\right), \quad L_{l}(s)=\frac{1}{2^{l} l !} \frac{d^{l}}{d s^{l}}\left[\left(s^{2}-1\right)^{l}\right]
$$


where $L_{l}$ is the Legendre polynomial. Then, replacing $\mathbf{u}(x, t)$ in Eq. with $\mathbf{u}_{h}(x, t)$ gives the ordinary differential equation (ODE):

$$
\begin{array}{r}
\frac{d}{d t} \mathbf{u}_{l}^{i}(t)-\frac{2 l+1}{\Delta_{i}}\left(\int_{I_{i}} \mathbf{f}\left(\mathbf{u}_{h}(x, t)\right)\left(\varphi_{l}^{i}(x)\right)_{x} d x-\hat{\mathbf{f}}\left(\mathbf{u}_{h}^{-}\left(x_{i+\frac{1}{2}}, t\right), \mathbf{u}_{h}^{+}\left(x_{i+\frac{1}{2}}, t\right)\right)\right. \\
\left.+(-1)^{l} \hat{\mathbf{f}}\left(\mathbf{u}_{h}^{-}\left(x_{i-\frac{1}{2}}, t\right), \mathbf{u}_{h}^{+}\left(x_{i-\frac{1}{2}}, t\right)\right)\right)=\frac{2 l+1}{\Delta_{i}} \int_{I_{i}} \mathbf{s}\left(\mathbf{u}_{h}(x, t)\right) \varphi_{l}^{i}(x) d x,
\end{array}
$$

which is used to solve the coefficients $\mathbf{u}_{l}^{i}(t)$. Here, the DG scheme features the assumption that the solution $\mathbf{u}_{h}$ is discontinuous at the cell boundaries. Thus, the flow values $\mathbf{f}\left(\mathbf{u}_{h}\left(x_{i \pm \frac{1}{2}}, t\right)\right)$ are replaced by the numerical fluxes $\hat{\mathbf{f}}\left(\mathbf{u}_{h}^{-}\left(x_{i \pm \frac{1}{2}}, t\right), \mathbf{u}_{h}^{+}\left(x_{i \pm \frac{1}{2}}, t\right)\right)$, where the function $\hat{\mathbf{f}}=\left(\hat{f}_{1}, \hat{f}_{2}\right)$ is defined by Eqs (18)-(22). The initial values of $\mathbf{u}_{l}^{i}(t)$ are derived by taking the equality between the integrations of $\mathbf{u}_{h}(x, 0) \varphi_{l}^{i}(x)$ and $\mathbf{u}(x, 0) \varphi_{l}^{i}(x)$ over $I_{i}$, which gives

$$
\mathbf{u}_{l}^{i}(0)=\frac{2 l+1}{\Delta_{i}} \int_{I_{i}} \mathbf{u}_{0}(x) \varphi_{l}^{i}(x) d x .
$$

Theoretically, the approximation is of the $(k+1)$-th order of accuracy. To ensure the same order of accuracy, all integrals are computed by the Gauss formula with sufficiently high accuracy (e.g., the two-point formula in the case of $k=1$ ).

\subsection{TVD Runge-Kutta time discretization}

Equations (24)-(25) can be denoted by the following ODEs:

$$
\left.\frac{d}{d t} \mathbf{u}_{h}\right|_{I_{i}}(t)=L_{h}\left(\mathbf{u}_{h}\right),\left.\quad \mathbf{u}_{h}\right|_{I_{i}}(0)=\sum_{l=0}^{k} \mathbf{u}_{l}^{i}(0) \varphi_{l}^{i}(x), i=1, \ldots, N,
$$

for which the $(k+1)$-th order TVD Runge-Kutta time discretization is adopted to ensure the same accuracy as that for the spatial discretization. Moreover, a slope limiter is used at each intermediate time step to ensure the TVD stability of the integral average of the solution over the cell. The procedure is briefed in the following.

For a division $\left\{t^{n}\right\}_{n=0}^{M}$ of the time interval $[0, t]$, where $t^{0}=0$, and $\Delta t^{n}=$ $t^{n+1}-t^{n}$, we set $\mathbf{u}_{i}^{0}=\Lambda \Pi_{h}^{k} \mathbf{u}_{i}(0)$. Then, for $n=0, \ldots, M-1, \mathbf{u}_{i}^{n+1}$ are computed as follows: 
1. Set $\mathbf{u}_{i}^{(0)}=\mathbf{u}_{i}^{n}$;

2. For $j=1, \ldots, k+1$, compute the intermediate functions:

$$
\mathbf{u}_{i}^{(j)}=\Lambda \Pi_{h}^{k}\left\{\Sigma_{l=0}^{j-1} \alpha_{j l} \mathbf{w}_{i}^{j l}\right\}, \quad \mathbf{w}_{i}^{j l}=\mathbf{u}_{i}^{(l)}+\frac{\beta_{j l}}{\alpha_{j l}} \Delta t^{n} L\left(\mathbf{u}_{i}^{(l)}\right) ;
$$

3. Set $\mathbf{u}_{i}^{n+1}=\mathbf{u}_{i}^{(k+1)}$.

The parameters $\alpha_{j l}$ and $\beta_{j l}$ are given in [4]. The slope limiter $\Lambda \Pi_{h}^{1}$, that acts on the piecewise linear solution $\left.\mathbf{v}_{h}\right|_{I_{i}}=\overline{\mathbf{v}}_{i}+\left(x-x_{i}\right) \mathbf{v}_{x, i}$, is defined by

$$
\left.\mathbf{u}_{h}\right|_{I_{i}}=\left.\Lambda \Pi_{h}^{1} \mathbf{v}_{h}\right|_{I_{i}}=\overline{\mathbf{v}}_{i}+\left(x-x_{i}\right) m\left(\mathbf{v}_{x, i}, \frac{\overline{\mathbf{v}}_{i+1}-\overline{\mathbf{v}}_{i}}{\Delta_{i} / 2}, \frac{\overline{\mathbf{v}}_{i}-\overline{\mathbf{v}}_{i-1}}{\Delta_{i} / 2}\right),
$$

where the minmod function

$$
m\left(a_{1}, a_{2}, a_{3}\right)=\left\{\begin{array}{l}
s \min _{1 \leq n \leq 3}\left|a_{n}\right|, \quad \text { ifs }=\operatorname{sign}\left(a_{1}\right)=\operatorname{sign}\left(a_{2}\right)=\operatorname{sign}\left(a_{3}\right), \\
0, \quad \text { otherwise. }
\end{array}\right.
$$

According to the definition of $\Lambda \Pi_{h}^{1}$, the limiter $\Lambda \Pi_{h}^{k}$ (for $k>1$ ) can be acquired from a standard procedure in [4].

We set $k=1$ for the simulation in the following section, which suggests that the scheme has second-order accuracy. See also $[1,27]$ for discussions of the DG scheme for other applications.

\section{Numerical simulation}

In system (3)-(4), we generally set the relaxation time as $\tau=30 \mathrm{~s}$, and the velocity-density relationships as

$$
\begin{gathered}
V(\rho)=v_{f} \frac{1-\rho / \rho_{\text {jam }}}{1+b\left(\rho / \rho_{\text {jam }}\right)+a\left(\rho / \rho_{\text {jam }}\right)^{2}}, \\
v_{e}(\rho)=v_{f}\left\{\left[1+\exp \left(\frac{\rho / \rho_{\text {jam }}-0.25}{0.06}\right)\right]^{-1}-3.72 \times 10^{-6}\right\},
\end{gathered}
$$

where $v_{f}=25 \mathrm{~m} / \mathrm{s}, a=4, b=-0.8$, and $\rho_{\text {jam }}=0.16 v e h . / \mathrm{m}$. Similar settings were chosen in $[30,19]$. To ensure the numerical stability of a scheme, the time step should satisfy the following CFL condition:

$$
\Delta t^{(n)}=C \frac{\Delta x}{\alpha^{(n)}},
$$


where $\alpha^{(n)}=\max _{i}\left\{\left|\lambda_{1}\left(\rho_{i}^{(n)}, w_{i}^{(n)}\right)\right|,\left|\lambda_{2}\left(\rho_{i}^{(n)}, w_{i}^{(n)}\right)\right|\right\}$.

The computational interval is set as $[0, L]$, with $L=16000 \mathrm{~m}$. However, the results shown in figures and tables are such that $x$ is scaled by $L, \rho$ and $w$ are scaled by $\rho_{j a m}$, and $t$ is scaled by $L / v_{f}$.

\subsection{Convergence to a smooth solution}

For system (3)-(4), there hardly exists a smooth and stable unsteady-state solution. The instability is due to the coupling effect between the "pressure" (or convection) and the "fluctuation" (or relaxation) in Eq. (4). Therefore, we remove the "fluctuation" and actually consider the homogeneous system (13) for deriving a smooth and stable solution.

Resorting to the discussion in the context of Eqs. (14)-(16), we assume that initially the profile of $\rho$ and $w$ is smooth and identical, given by

$$
w(x, 0)=\rho(x, 0)=\frac{1}{4}-\frac{1}{10} \sin (2 \pi x)
$$

This suggests that $z(x, 0)=1$, and thus $z(x, t)=1$ solves Eq. (16) exactly. This solution is related to a linearly degenerate characteristic field, and suggests that $\rho(x, t)=w(x, t)$. For Eq. (14), which is related to a genuinely nonlinear characteristic field, the solution $w(x, t)$ is derived by parallelly moving the initial profile of $w(x, 0)$ a distance $\lambda_{1}(w(x, 0)) t$ along $x$ axis, thus we have

$$
w(x, t)=\rho(x, t)=w\left(x-\lambda_{1}(w(x, 0)) t, 0\right)
$$

This solution is smooth and well-posed before a distorted profile appears, whereafter a discontinuity (shock) should be introduced. See [25, 24] for details of the related theory.

We confirm that, for the initial conditions (26), Eq. (27) suggests a wellposed smooth solution at $t=50 \mathrm{~s}$, which is before the appearance of a distorted profile or shock. This solution is used for convergence test. Besides, it provides the boundary conditions for computation. The test achieves almost the same order of accuracy as that theoretically predicted in the discussed DG scheme, for $k=1$ and $k=2$, and for applications of all extended Riemann solvers. Table 1 shows the results given by the EO and TF numerical fluxes. 
Table $1 L_{1}(0,1)$ and $L_{\infty}(0,1)$ errors and orders of accuracy for convergence of $w(x, t)$ in the DG scheme, where the exact smooth solution is given by Eqs. (26)-(27).

\begin{tabular}{|c|c|c|c|c|c|c|c|c|c|}
\hline \multirow{8}{*}{ EO flux } & \multicolumn{5}{|c|}{$k=1 \quad C=0.3$} & \multicolumn{4}{|c|}{$k=2 \quad C=0.2$} \\
\hline & $\mathrm{N}$ & $L_{1}$ error & order & $L_{\infty}$ error & order & $L_{1}$ error & order & $L_{\infty}$ error & order \\
\hline & 20 & $2.75 \mathrm{E}-04$ & & $4.55 \mathrm{E}-04$ & & $6.87 \mathrm{E}-06$ & & $2.23 \mathrm{E}-05$ & \\
\hline & 40 & $6.80 \mathrm{E}-05$ & 2.02 & $1.12 \mathrm{E}-04$ & 2.02 & $9.66 \mathrm{E}-07$ & 2.83 & $3.33 \mathrm{E}-06$ & 2.74 \\
\hline & 80 & $1.70 \mathrm{E}-05$ & 2.00 & $2.77 \mathrm{E}-05$ & 2.01 & $1.33 \mathrm{E}-07$ & 2.86 & $4.71 \mathrm{E}-07$ & 2.82 \\
\hline & 160 & $4.24 \mathrm{E}-06$ & 2.00 & $6.96 \mathrm{E}-06$ & 2.00 & $1.77 \mathrm{E}-08$ & 2.92 & $6.16 \mathrm{E}-08$ & 2.94 \\
\hline & 320 & $1.06 \mathrm{E}-06$ & 2.00 & $1.75 \mathrm{E}-06$ & 1.99 & $2.24 \mathrm{E}-09$ & 2.98 & 7.94E-09 & 2.96 \\
\hline & 640 & $2.65 \mathrm{E}-07$ & 2.00 & $4.39 \mathrm{E}-07$ & 2.00 & $2.81 \mathrm{E}-10$ & 3.00 & $1.05 \mathrm{E}-09$ & 2.91 \\
\hline \multirow{8}{*}{ TF flux } & & & $k=1$ & $C=0.25$ & & & $k=2$ & $C=0.15$ & \\
\hline & $\mathrm{N}$ & $L_{1}$ error & order & $L_{\infty}$ error & order & $L_{1}$ error & order & $L_{\infty}$ error & order \\
\hline & 20 & $2.74 \mathrm{E}-04$ & & $4.54 \mathrm{E}-04$ & & $1.41 \mathrm{E}-05$ & & $4.35 \mathrm{E}-05$ & \\
\hline & 40 & $6.79 \mathrm{E}-05$ & 2.01 & $1.13 \mathrm{E}-04$ & 2.01 & $2.50 \mathrm{E}-06$ & 2.50 & $8.59 \mathrm{E}-06$ & 2.34 \\
\hline & 80 & $1.70 \mathrm{E}-05$ & 2.00 & $2.82 \mathrm{E}-05$ & 2.00 & $4.08 \mathrm{E}-07$ & 2.62 & $1.49 \mathrm{E}-06$ & 2.53 \\
\hline & 160 & $4.24 \mathrm{E}-06$ & 2.00 & $7.03 \mathrm{E}-06$ & 2.00 & $6.44 \mathrm{E}-08$ & 2.66 & $2.33 \mathrm{E}-07$ & 2.68 \\
\hline & 320 & $1.06 \mathrm{E}-06$ & 2.00 & $1.76 \mathrm{E}-06$ & 2.00 & $1.00 \mathrm{E}-08$ & 2.68 & $3.37 \mathrm{E}-08$ & 2.79 \\
\hline & 640 & $2.65 \mathrm{E}-07$ & 2.00 & 4.39E-07 & 2.00 & $1.55 \mathrm{E}-09$ & 2.69 & $5.26 \mathrm{E}-09$ & 2.68 \\
\hline
\end{tabular}

\subsection{Convergence to a wide moving jam solution with discontinuity}

The periodic boundary conditions are applied, and the initial values are set as

$$
\left\{\begin{array}{l}
\rho(x, 0)=\rho_{0}+\Delta \rho_{0}\left\{\cosh ^{-2}\left[\frac{160}{L}\left(x-\frac{3 L}{8}\right)\right]-\frac{1}{4} \cosh ^{-2}\left[\frac{40}{L}\left(x-\frac{13 L}{32}\right)\right]\right\} \\
w(x, 0)=V^{-1}\left(v_{e}(\rho(x, 0))\right)
\end{array}\right.
$$

We set $\Delta x=10 \mathrm{~m}$ in all schemes. The constant $C$ in the CFL condition is set as follows: $C=0.5$ in the second-order accurate DG scheme, $C=0.2$ in the third-order accurate DG scheme except that $C=0.1$ for the TF flux; $C=1.0$ in the first-order accurate and the fifth-order accurate WENO schemes, except that $C=0.68$ for the TF flux.

(a)
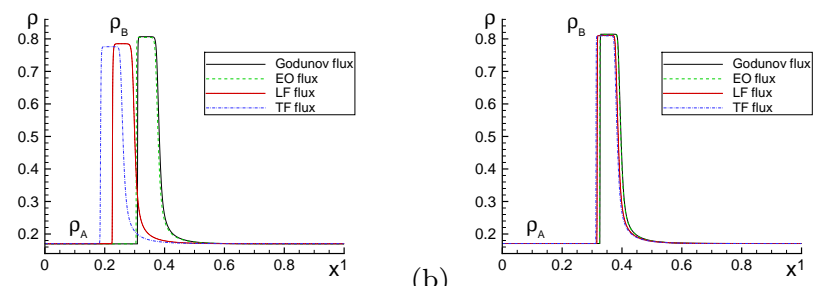

(c)

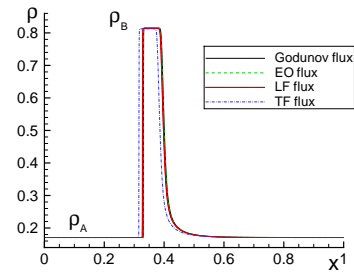

Fig. 3 Comparison of the numerical results $(t=5600 \mathrm{~s})$. (a) The first-order finite volume scheme. (b) The fifth-order WENO finite volume scheme. (c) The second-order DG scheme. 
Equation (28) can be viewed as a perturbed state from the equilibrium $\left(\rho_{0}, w_{0}\right)$, where $w_{0}=V^{-1}\left(v_{e}\left(\rho_{0}\right)\right)$. According to Whitham [25], such an equilibrium solution is linearly stable if the so-called kinetic wave speed $q_{e}^{\prime}\left(\rho_{0}\right)$ is between the two characteristic speeds $\lambda_{1}\left(w_{0}\right)$ and $\lambda_{2}\left(w_{0}\right)$. We use this argument to determine two critical densities, $\rho_{c_{1}}=0.1113 \rho_{j a m}$ and $\rho_{c_{1}}=$ $0.4240 \rho_{\text {jam }}$, such that the equilibrium is linearly stable for $\rho_{0} \leq \rho_{c_{1}}$ or $\rho_{0} \geq \rho_{c_{2}}$ and unstable for $\rho_{0} \in\left(\rho_{c_{1}}, \rho_{c_{2}}\right)$. This means that the perturbed state of (28) is expected to regress to the equilibrium state if $\rho_{0} \leq \rho_{c_{1}}$ or $\rho_{0} \geq \rho_{c_{2}}$; otherwise, it is very likely to evolve into stop-and-go waves, according to the discussions in $[10,31,32,7,22]$ (especially in [28]). The mechanism for the stability and instability of a phase state may also be more profoundly associated with the physically bounded solutions of the model [33].

Table 2 Comparison between the characteristic parameters of a wide moving jam acquired by the numerical and analytical solutions; $\rho_{A}$ and $\rho_{B}$ denote the minimal and maximal densities of the solution.

\begin{tabular}{ccccccccc}
\hline & \multicolumn{2}{c}{ Godunov flux } & \multicolumn{2}{c}{ EO flux } & \multicolumn{2}{c}{ LF flux } & \multicolumn{2}{c}{ TF flux } \\
\cline { 2 - 9 } & $\rho_{A}$ & $\rho_{B}$ & $\rho_{A}$ & $\rho_{B}$ & $\rho_{A}$ & $\rho_{B}$ & $\rho_{A}$ & $\rho_{B}$ \\
\hline First-order scheme & 0.1697 & 0.8067 & 0.1697 & 0.8046 & 0.1702 & 0.7848 & 0.1703 & 0.7759 \\
WENO scheme & 0.1708 & 0.8143 & 0.1708 & 0.8140 & 0.1708 & 0.8105 & 0.1708 & 0.8093 \\
DG scheme (k=1) & 0.1708 & 0.8152 & 0.1708 & 0.8148 & 0.1708 & 0.8139 & 0.1707 & 0.8124 \\
DG scheme (k=2) & 0.1708 & 0.8166 & 0.1708 & 0.8163 & 0.1708 & 0.8155 & 0.1708 & 0.8141 \\
\hline Analytical results & \multicolumn{3}{c}{$\rho_{A}=0.1708$} & \multicolumn{4}{c}{$\rho_{B}=0.8267$} \\
\hline
\end{tabular}

We choose $\rho_{0}=0.22 \rho_{\text {jam }} \in\left(\rho_{c_{1}}, \rho_{c_{2}}\right)$ and $\Delta \rho_{0}=0.2 \rho_{\text {jam }}$, where $\rho_{0}$ is sufficiently large that we expect an evolution into a wide moving jam. As Fig. 3 shows, we observe the wide moving jam reproduced by the first-order accurate, the fifth-order accurate WENO and the second-order accurate DG schemes, all of which are combined with the extended Godunov, EO, LF and TF numerical fluxes defined by Eqs (18)-(22). In Table 2, the characteristic parameters (the maximal and minimal densities) of these simulated solutions are also compared with those acquired from the discussion in Section 2.2.

When this sequence is followed, the extended numerical fluxes become simpler and the computational cost is reduced. In general, these results agree well with the analytical findings determined by Eq. (11). However, the numerical viscosities or errors increase when these fluxes are applied in the first-order accurate scheme, as shown in Fig 3(a). In contrast, the differences between these fluxes in generating numerical viscosity are considerably sup- 
pressed in the fifth-order accurate WENO and the second-order DG schemes, as shown in Figs. 3(b) and (c).

Table 3 Comparison between the fifth-order accurate WENO and the DG schemes in generating errors, with the first-order accuracy shown for convergence to the analytical result. The Godunov flux is adopted for both schemes.

\begin{tabular}{c|ccccc}
\hline & $\begin{array}{c}\text { Space step } \\
(\Delta x=10 m)\end{array}$ & $\rho_{A}$ & $\rho_{B}$ & $\begin{array}{c}\text { Error } \\
\text { in } \rho_{B}\end{array}$ & $\begin{array}{c}\text { Convergence order } \\
\text { in } \rho_{B}\end{array}$ \\
\hline & $\Delta x$ & 0.1708 & 0.8143 & 0.0124 & \\
WENO scheme & $\Delta x / 2$ & 0.1708 & 0.8204 & 0.0063 & 0.9767 \\
& $\Delta x / 4$ & 0.1708 & 0.8236 & 0.0031 & 1.0231 \\
& $\Delta x / 8$ & 0.1708 & 0.8251 & 0.0016 & 0.9542 \\
& $\Delta x / 16$ & 0.1708 & 0.8258 & 0.0009 & 0.8301 \\
\hline \multirow{5}{*}{ DG scheme $(k=1)$} & $\Delta x$ & 0.1708 & 0.8152 & 0.0115 & \\
& $\Delta x / 2$ & 0.1708 & 0.8209 & 0.0058 & 0.9875 \\
& $\Delta x / 4$ & 0.1708 & 0.8237 & 0.0030 & 0.9511 \\
& $\Delta x / 8$ & 0.1708 & 0.8252 & 0.0015 & 1.0000 \\
& $\Delta x / 16$ & 0.1708 & 0.8258 & 0.0009 & 0.7370 \\
\hline & $\Delta x$ & 0.1708 & 0.8166 & 0.0101 & \\
& $\Delta x / 2$ & 0.1708 & 0.8216 & 0.0051 & 0.9858 \\
& $\Delta x / 4$ & 0.1708 & 0.8241 & 0.0026 & 0.9702 \\
& $\Delta x / 8$ & 0.1708 & 0.8253 & 0.0014 & 0.8931 \\
& $\Delta x / 16$ & 0.1708 & 0.8259 & 0.0008 & 0.8074 \\
\hline
\end{tabular}

Figs. 3(b) and (c), together with Table 2, indicate that the DG and WENO schemes produce similar numerical results even though the theoretical accuracy of the latter scheme is higher than that of the former scheme. To enhance the conclusion, the errors generated by the two schemes are indicated in Table 3 for comparison. With refined grids, Table 3 also shows a first-order accurate convergence to the analytical result for these schemes. We note that the reduction in the order of accuracy is due to the shock discontinuity in the solution profile. This indicates the efficiency of the second-order accurate DG scheme in resolving the profile of a wide moving jam in traffic flow.

\section{Conclusions}

The CHO model is composed of the mass and pseudo-mass conservations, which are favorably consistent with the LWR model. Underlying this consistency is the finding that the Riemann solver for the model can be expressed 
by the classical Godunov flux, which is based on a Riemann invariant that remains unchanged through propagation from the initial state on the upstream side to the interface. Accordingly, the classical EO and LF fluxes, together with a TF flux, are extended, allowing us to obtain a number of Riemann solvers for solving the CHO model. In general, we find that these fluxes work well in generating stable and convergent solutions when applied in both the first-order scheme and the higher-order schemes.

In particular, we conclude that the second-order accurate DG scheme is efficient for convergence to a traveling wave solution with a shock profile, especially when combined with a simpler Riemann solver. In fact, the scheme matches the third-order accurate DG and the fifth-order WENO scheme in suppressing the errors or numerical viscosities between all of the extended fluxes (Tables 2 and 3); these errors are clearly shown in the results generated by the first-order accurate schemes (Fig. 3). The conclusion might also be suited for other second-order accurate total variation diminishing (TVD) schemes.

The simulated wide moving jam solution together with the evolution reflects many typical phenomena in traffic. Therefore, the indicated efficiency is significant when the model is extended to simulate traffic flow on a road network, in which case much greater computational resources is required. The extension of the model and the corresponding numerical scheme will be discussed in our future works.

\section{Acknowledgements}

This study was jointly supported by grants from the National Natural Science Foundation of China $(11072141,11272199)$, the National Basic Research Program of China (2012CB725404), the Shanghai Program for Innovative Research Team in Universities, and a National Research Foundation of Korea grant funded by the Korean government (MSIP) (NRF-2010-0029446).

\section{References}

[1] F.Bassi, and S. Rebay, A high-order accurate discontinuous finite element method for the numerical solution of the compressible NavierCStokes equations. J. Comput. Phys. 131 (1997) 267-279.

[2] R. Bürger, R. Donat, P. Mulet, C.A. Vega, Hyperbolicity analysis of polydisperse sedimentation models via a secular equation for the flux Jacobian, SIAM Journal on Applied Mathematics. 70(7) (2010) 2186-2213. 
[3] R. Bürger, A. García, K.H. Karlsen, J.D. Towers, A family of numerical schemes for kinematic flows with discontinuous flux, Journal of Engineering Mathematics. 60(3-4) (2008) 387-425.

[4] B. Cockburn, C.-W. Shu, Runge-Kutta discontinuous Galerkin methods for convection-dominated problems, Journal of Scientific Computing. 16(3) (2001) 173-261.

[5] R. Donat, P. Mulet, Characteristic-based schemes for multi-class LighthillWhitham-Richards traffic models, Journal of Scientific Computing. 37(3) (2008) 233-250.

[6] M. Dumbser and E.F. Toro. On Universal Osher-Type Schemes for General Nonlinear Hyperbolic Conservation Laws, Communications in Computational Physics. 10(3) (2011) 635-671.

[7] J.M. Greenberg, Congestion redux, SIAM J. Appl. Math. 64(4) (2004) 11751185 .

[8] W.L. Jin, H.M. Zhang, The inhomogeneous kinematic wave traffic flow model as a resonant nonlinear system, Transportation Science. 37(3) (2003) 294311.

[9] W.L. Jin, H.M. Zhang, On the distribution schemes for determining flows through a merge, Transportation Research Part B. 37(6) (2003) 521-540.

[10] B.S. Kerner, P. Konhauser, Structure and parameters of clusters in traffic flow, Phys. Rev. E. 50 (1994) 54-83.

[11] F. van Wageningen-Kessels, B. vant Hof, S.P. Hoogendoorn, H. vant Lint, K. Vuik, Anisotropy in generic multi-class traffic flow models, Transportmetrica A: Transpor Science. 9(5) (2013) 451-472.

[12] E.J. Kubatko, J.J. Westerink, C. Dawson. Semi discrete discontinuous Galerkin methods and stage-exceeding-order, strong-stability-preserving RungeCKutta time discretizations,Journal of Computational Physics, $222(2007) 832-848$.

[13] J.P. Lebacque, S. Mammar, and H. Haj-Salem, The Aw-Rascle and Zhang's model: vacuum problems, existence and regularity of the solutions of the Riemann problem. Transportation Research Part B, 41(7) (2007), 710-721.

[14] J.P. Lebacque, S. Mammar, and H. Haj-Salem, Generic second order traffic flow modelling. In R.E. Allsop, M.G.H. Bell, and B.G. Heydecker (eds.), Transportation and Traffic Theory, Elsevier, London, (2007) 755-776.

[15] J. Li, Q.Y. Chen, H.Z. Wang, D.H. Ni, Analysis of LWR model with fundamental diagram subject to uncertainties, Transportmetrica. 8(6) (2012) 387-405. 
[16] M.J. Lighthill, G.B. Whitham, On kinematic waves. II. A theory of traffic flow on long crowded roads, Proceedings of the Royal Society of London. Series A, Mathematical and Physical Sciences. (1955) 317-345.

[17] D. Ngoduy, Platoon-based macroscopic model for intelligent traffic flow, Transportmetrica B: Transport Dynamics. 1(2)(2013) 153-169.

[18] H.J. Payne, Models of freeway traffic and control, Mathematical Models of Public Systems. 1(1) (1971) 51-61.

[19] D.L. Qiao, P. Zhang, Extended numerical fluxes and the corresponding WENO schemes for a conserved higher-order traffic flow model (in Chinese), Proceedings of celebrating Professor Dai Shiqiang's 70 birthday cum 50 years engaged in scientific research. Shanghai: Shanghai University Press (2011).

[20] M. Rascle, An improved macroscopic model of traffic flow: Derivation and links with the Lighthill-Whitham model, Math. Comput. Modelling. 35 (2002) 581-590.

[21] P.I. Richards, Shock waves on the highway, Operations Research. 4 (1956) $42-51$.

[22] F. Siebel, W. Mauser, On the fundamental diagram of traffic flow. SIAM Journal of Applied Mathematics. 66 (2006) 1150-1162.

[23] C.-W. Shu, High order weighted essentially nonoscillatory schemes for convection dominated problems, SIAM Rev. 51(1) (2009) 82-126.

[24] E.F. Toro, Riemann solvers and numerical methods for fluid dynamics: a practical introduction, Berlin: Springer Verlag. (2009)

[25] G.B. Whitham, Linear and nonlinear waves, New York: Wiley New York. 226 (1974).

[26] J.K. Wiens, J.M. Stockie, J.F. Williams, Riemann solver for a kinematic wave traffic model with discontinuous flux, Journal of Computational Physics. 242 (2013) 1-23.

[27] Y. Xu, C.-W. Shu, Local discontinuous Galerkin methods for high-order time-dependent partial differential equations, Commun. Comput. Phys. 7(1) (2010) $1-46$.

[28] R.Y. Xu, P. Zhang, S.Q. Dai, S.C. Wong, Admissibility of a wide cluster solution in "anisotropic" higher-order traffic flow models, SIAM J. Appl. Math. 68 (2007) 562-573.

[29] P. Zhang, R.X. Liu, S.C. Wong, S.Q. Dai, Hyperbolicity and kinematic waves of a class of multi-population partial differential equations, European Journal of Applied Mathematics. 17 (2006) 171-200. 
[30] P. Zhang, D.L. Qiao, L.Y. Dong, S.Q. Dai, S.C. Wong, A number of Riemann solvers for a conserved higher order traffic flow model, Fourth International Joint Conference on Computational Sciences and Optimization (CSO), Lijiang: IEEE. (2011) 1049-1053.

[31] P. Zhang, S.C. Wong, S.Q. Dai, Characteristic parameters of a wide cluster in a higher-order traffic flow model, Chinese Physics Letters. 23 (2006) 516519 .

[32] P. Zhang, S.C. Wong, Essence of conservation forms in the traveling wave solutions of higher-order traffic flow models, Phys. Rev. E. 74 (2006) 0261091-026109-10.

[33] P. Zhang, S.C. Wong, S.Q. Dai, A conserved higher-order anisotropic traffic flow model: Description of equilibrium and non-equilibrium flows, Transportation Research Part B: Methodological. 43(5) (2009) 562-574.

[34] P. Zhang, C.X. Wu, S.C. Wong, A semi-discrete model and its approach to a solution for wide moving jam in traffic flow, Physica A. 391(3) (2012) 456-463.

[35] R.X. Zhong, A. Sumalee, T.L. Pan, and W.H.K. Lam, Stochastic cell transmission model for traffic network with demand and supply uncertainties. Transportmetrica A: Transport Science. 9(7)(2013) 567-602. 